\title{
Control químico de Moniliophthora roreri en México
}

\author{
Chemical control of Moniliophthora roreri in Mexico
}

\begin{abstract}
Magdiel Torres-de-la-Cruz', Isaí Quevedo-Damián², Carlos Fredy Ortiz-García*, Luz del Carmen Lagúnez-Espinoza², Daniel Nieto-Angel ${ }^{3}$, Manuel Pérez-de la Cruz ${ }^{1}$

División Académica de Ciencias Biológicas, Universidad Juárez Autónoma de Tabasco. Km. 0.5 Carretera VillahermosaCárdenas, Villahermosa, Centro, Tabasco, México.

Colegio de Postgraduados, Campus Tabasco. Km. 3.5 Carretera Cárdenas-Huimanguillo, H. Cárdenas, Tabasco, México. CP 86500.

3 Colegio de Postgraduados, Campus Montecillo. km 36.5 Carretera México-Texcoco, Texcoco, Estado de México, México. CP 56230.
\end{abstract}

\section{RESUMEN}

Moniliophthora roreri causa la moniliasis (MC), la enfermedad fúngica más destructiva del fruto del cacao en América, con pérdidas hasta del $100 \%$. En México, el empleo del control químico de la MC es escaso, debido principalmente a la falta de productos seleccionados por su efectividad sobre $M$. roreri. El objetivo fue evaluar la eficacia de fungicidas sistémicos y protectantes sobre $M$. roreri. In vitro, se evaluaron cinco fungicidas sistémicos y siete protectantes. In vivo, se evaluó una aplicación de los fungicidas sistémicos tebuconazol, propiconazol, azoxystrobin y trifloxystrobin a 0.4 y 0.8 g.i.a./L, sobre frutos juveniles de dos meses de edad; complementada con tres aplicaciones de hidróxido de cobre a 7.5 g.i.a./L. In vitro, M. roreri fue sensible a los fungicidas sistémicos azoxystrobin, trifloxystrobin, tebuconazole y propiconazole. También fue sensible a los fungicidas protectantes: sulfato de cobre, hidróxido de cobre, óxido cuproso, oxicloruro de cobre y polisulfuro de calcio. In vivo, una aplicación de azoxystrobin, trifloxystrobin, tebuconazol o propiconazol (0.8 g.i.a./L), complementada con hidróxido de cobre, disminuyó significativamente la incidencia de la MC. La sensibilidad in vitro de M. roreri, al sulfato de cobre, oxido cuproso y oxicloruro de cobre, abre la posibilidad de probarlos en campo en futuras investigaciones.

Palabras clave: Control químico, moniliasis, Theobroma cacao, fungicidas.

\section{ABSTRACT}

Moniliophthora roreri cause the frosty pod rot (FPR), the most destructive fungal disease of the cacao fruit in America, with losses of up to $100 \%$. In Mexico, the chemical control of the FPR has been scarce, mainly due to the lack of selected products for its effectiveness on $M$. roreri. The aim was to evaluate the efficacy of systemic and protectant fungicides on M. roreri. In vitro, five systemic fungicides and seven protectants were evaluated. In vivo, we assessed an application of the systemic fungicides tebuconazole, propiconazole, azoxystrobin and trifloxystrobin at 0.4 and 0.8 g.a.i./L, on two-months age fruits; complemented with three applications of copper hydroxide at 7.5 g.a.i./L. In vitro, M. roreri was

*Autor para correspondencia: Carlos Fredy Ortiz García

Correo electrónico: cfortizg@gmail.com

Recibido: 29 de mayo de 2018

Aceptado: 30 de octubre de 2018 sensitive to azoxystrobin, trifloxystrobin, tebuconazole and propiconazole systemic fungicides. Also, it was sensitive to copper sulphate, copper hydroxide, cuprous oxide, copper oxychloride and calcium polysulfide protectant fungicides. In vivo, an application of azoxystrobin, trifloxystrobin, tebuconazole or propiconazole (0.8 g.a.i./L), supplemented with copper hydroxide, significantly decreased the incidence of FPR. The in vitro sensitivity of $M$. roreri, to the copper sulphate, cuprous oxide and copper oxychloride, opens the possibility of testing them in the field, in future research.

Key words: Chemical control, Frosty pod rot, Theobroma cacao, fungicides.

\section{INTRODUCCIÓN}

El basidiomycete Moniliophthora roreri causa la moniliasis del cacao (MC), la enfermedad más limitante de la producción de Theobroma cacao en el continente americano. Las pérdidas ocasionadas por este patógeno en México son superiores al $75 \%$ de la producción (Torres-de la Cruz et al., 2011), con consecuencias socioeconómicas y ambientales que pone en riesgo la sustentabilidad del cultivo de cacao.

Moniliophthora roreri ataca frutos en diferentes estados de desarrollo; sin embargo, éstos son más susceptibles en etapas juveniles (Ampuero, 1967). Posee un ciclo de vida hemibiotrófico (Griffith et al., 2003), por lo que los frutos pueden estar infectados sin mostrar daños internos y externos. Los únicos propágulos infectivos son las esporas, las cuales germinan y colonizan el endocarpo de forma intercelular. Posteriormente, el hongo invade los tejidos del mesocarpo y exocarpo de forma intracelular, dando lugar a la necrosis (Amaya et al., 1976). En la superficie del fruto, las manchas necróticas se cubren de micelio y abundante esporulación; de esta manera, $M$. roreri completa su ciclo de vida.

En el combate de la MC, el control cultural ha sido el método más utilizado, el cual ha incluido el control de malezas, mejoramiento del drenaje, poda del árbol de cacao y de los árboles de sombra (Barros, 1966; Galindo, 1984). Así también, la eliminación total de frutos en periodo de baja o nula producción, cosecha oportuna (Bateman et al., 2005) y la remoción semanal de frutos enfermos (Krauss et al., 
2003). Otros autores han recomendado el uso de micoparásitos como agentes de control biológico (Krauss y Soberanis, 2002). Por otra parte, Phillips-Mora et al. (2005) seleccionaron el clon ICS-95, con resistencia contra M. roreri; sin embargo, el éxito del control genético sobre la MC aún no se ve reflejado a escala comercial.

En el control químico de la MC, en Centro y Suramérica se han obtenido resultados promisorios con fungicidas basados en cobre (óxido cuproso, hidróxido de cobre) y con el fungicida sistémico flutolanil (González et al., 1983; Hidalgo et al., 2003; Bateman et al., 2005). En México, el control químico ha sido una práctica poco empleada, debido principalmente a la poca disponibilidad de productos seleccionados por su efectividad sobre $M$. roreri.

Actualmente, en el mercado nacional existen diversos fungicidas protectantes basados en diferentes sales de cobre (cúpricos), los cuales, por su eficiencia, bajo costo y bajo impacto ambiental, representan una opción viable de ser evaluada. Así también, existen fungicidas sistémicos con capacidad de translocación y con efecto erradicativo de infecciones recién establecidas, los cuales pueden ser utilizados en etapas tempranas de desarrollo de la epidemia (Bateman et al. 2005). Estos fungicidas pueden ser evaluados contra $M$. roreri e incluirse en programas de manejo de la MC. Así, el objetivo de este trabajo fue evaluar la eficacia de fungicidas sistémicos y protectantes in vitro e in vivo sobre M. roreri, agente causal de la MC en México.

\section{MATERIALES Y MÉTODOS}

\section{Obtención del aislamiento de Moniliophthora roreri}

El aislamiento de $M$. roreri fue adquirido en el Laboratorio de Fitopatología del Colegio de Postgraduados Campus Tabasco. Este aislamiento se identificó por morfología y secuencia de ITS (GenBank: GU108605) por Torres-de la Cruz et al. (2016).

\section{Bioensayos de sensibilidad in vitro}

Cinco fungicidas sistémicos y siete fungicidas protectantes se evaluaron sobre la germinación de esporas (GE) y el crecimiento micelial (CM) de M. roreri. Cada fungicida se agregó al medio agar dextrosa y papa (ADP) (Difco Laboratories, Detroit, MI, USA) antes de vaciar en las cajas de Petri. El polisulfuro de calcio se elaboró de acuerdo con Ramírez et al. (2011). Cada fungicida se evaluó en cuatro dosis: la dosis recomendada o utilizada y tres dosis inferiores. Esta prueba se realizó por duplicado. Los fungicidas y sus respectivas concentraciones se muestran en la Tabla 1.

\section{Efectividad de fungicidas in vitro, sobre la germinación de esporas de $M$. roreri}

Cultivos de M. roreri de $10 \mathrm{~d}$ se utilizaron para obtener una suspensión de $5 \times 10^{6}$ esporas $/ \mathrm{mL}$. De esta suspensión, 30 $\mu \mathrm{L}$ se depositaron en cuatro zonas de la caja de Petri con medio ADP, con las concentraciones de fungicidas mostradas en la Tabla 1. La alícuota de esporas se cubrió con cubreobjetos. Las cajas con medio ADP + inóculo se incubaron a $25 \pm 0.5^{\circ} \mathrm{C}$. El porcentaje de GE se determinó mediante la observación de 100 esporas por tratamiento y repetición. Las observaciones se efectuaron cada $2 \mathrm{~h}$ (el primer día), después, cada 24 h y concluyeron cuando el testigo obtuvo el $90 \%$ de germinación.

\section{Efectividad de fungicidas in vitro, sobre el crecimiento micelial de $M$. roreri}

A partir de cultivos de $M$. roreri de $15 \mathrm{~d}$ de edad, fragmentos de $5 \mathrm{~mm}$ de diámetro se extrajeron con un sacabocado. Estas fracciones se depositaron en el centro de cajas

Tabla 1. Fungicidas y dosis evaluadas in vitro sobre Moniliophthora roreri.

Table 1. Fungicides and doses evaluated in vitro on Moniliophthora roreri.

\begin{tabular}{llcccc}
\hline Fungicida & $\begin{array}{c}\text { Modo de } \\
\text { acción }\end{array}$ & \multicolumn{5}{c}{ Dosis (g.i.a./L) } \\
\cline { 3 - 6 } & & $\mathbf{1}$ & $\mathbf{2}$ & $\mathbf{3}$ & $\mathbf{4}$ \\
\hline Sulfato de cobre 250 & Protectante & 0.62 & 1.25 & 1.87 & 2.5 \\
\hline Hidróxido cúprico & Protectante & 1.87 & 3.75 & 5.62 & 7.5 \\
\hline Óxido cuproso & Protectante & 0.93 & 1.87 & 2.81 & 3.75 \\
\hline Oxicloruro de cobre (OC) & Protectante & 1.25 & 2.5 & 3.75 & 5 \\
\hline Polisulfuro de calcio & Protectante & 4 & 8 & 12 & 16 \\
\hline Azufre elemental + OC & Protectante & $0.45 / 0.04$ & $0.9 / 0.08$ & $1.35 / 0.12$ & $1.8 / 0.16$ \\
\hline Clorotalonil & Protectante & 0.25 & 0.5 & 0.75 & 1 \\
\hline Azoxystrobin & Sistémicoy & 0.25 & 0.5 & 0.75 & 1 \\
\hline Trifloxystrobin & Sistémico & 0.25 & 0.5 & 0.75 & 1 \\
\hline Tebuconazole & Sistémico & 0.25 & 0.5 & 0.75 & 1 \\
\hline Propiconazole & Sistémico & 0.25 & 0.5 & 0.75 & 1 \\
\hline Tiabendazol & Sistémico & 0.25 & 0.5 & 0.75 & 1 \\
\hline
\end{tabular}

'De acción translaminar

${ }^{z}$ g.i.a. $=$ gramos de ingrediente activo 
de Petri con medio ADP, las cuales contenían las concentraciones de fungicidas mostradas en la Tabla 1. Las cajas con medio e inóculo se incubaron a $25 \pm 0.5^{\circ} \mathrm{C}$. El CM, medido en dos radios por caja, se registró cada $24 \mathrm{~h}$ y las mediciones concluyeron cuando el testigo llenó la caja de Petri.

\section{Efecto de fungicidas sobre la moniliasis del cacao in vivo}

En el municipio de Jalpa de Méndez, Tabasco, se seleccionaron dos plantaciones de cacao de 22 años de edad; ubicadas a $13 \mathrm{msnm}, 18^{\circ} 10^{\prime} 33.5^{\prime \prime} \mathrm{N}, 93^{\circ} 08^{\prime} 45^{\prime \prime} \mathrm{O}$ y $15 \mathrm{msnm}$, $18^{\circ} 9^{\prime} 34^{\prime \prime} \mathrm{N}, 93^{\circ} 9^{\prime} 10.5^{\prime \prime} \mathrm{O}$, respectivamente. Las plantaciones estuvieron constituidas por el complejo Trinitario mexicano, susceptibles a M. roreri, establecidos por semilla y dispuestos en un arreglo espacial de $3.5 \times 3.5 \mathrm{~m}$. En cada parcela, 45 árboles de cacao fueron seleccionados, y en cada árbol, 10 frutos de dos meses de edad fueron marcados. Cada árbol con sus respectivos frutos se consideró una unidad experimental (repetición). Los frutos fueron etiquetados con una clave respectiva al tratamiento.

Con base en los resultados de sensibilidad in vitro, se evaluó la efectividad de una aplicación de los fungicidas sistémicos tebuconazol, propiconazol, azoxystrobin y trifloxystrobin a 0.4 y 0.8 g.i.a./L, sobre frutos de dos meses de edad; complementado con tres aplicaciones posteriores, a intervalo mensual, de hidróxido de cobre (protectante) a 7.5 g.i.a./L, de acuerdo con Bateman et al. (2005). Así, ocho tratamientos más un testigo, fueron establecidos. Todos los fungicidas fueron aplicados mediante aspersión dirigida a los frutos, utilizando bomba de mochila. El número de frutos que manifestaron síntomas externos de la MC (manchas necróticas y esporulación) se cuantificó semanalmente, de acuerdo con Torres-de la Cruz et al. (2011) y se calculó la incidencia semanal.

\section{Análisis estadístico}

Todos los ensayos se analizaron bajo un diseño completamente al azar, con cinco repeticiones. In vivo, cada réplica consistió en un árbol de cacao con 10 frutos de dos meses de edad. La efectividad de los fungicidas evaluados (in vitro e in vivo), se obtuvo de acuerdo con Abbott (1925). Posteriormente, los datos se transformaron al arcoseno de la raíz cuadrada de la proporción y se sometieron a un ANOVA y separación de medias (Tukey; $\mathrm{P} \leq 0.05$ ) utilizando el programa SAS (SAS, 1988).

\section{RESULTADOS}

\section{Efectividad de fungicidas in vitro}

Hubo diferencias en la efectividad de fungicidas sistémicos sobre el CM y la GE de M. roreri $(\mathrm{P}<0.0001)$. El trifloxystrobin, el tebuconazol y el propiconazol inhibieron el $100 \%$ del CM en todas las dosis evaluadas; el azoxystrobin mostró una eficiencia de 72.4 al $78.7 \%$. El fungicida sistémico con menor efectividad sobre el CM fue el tiabendazol (Tabla 2). En el testigo (sin fungicida), la GE promedio fue $98 \%$ después de las $96 \mathrm{~h}$; sin embargo, el trifloxystrobin y el tebuconazole inhibieron la GE al $100 \%$ en todas las dosis evaluadas. El
Tabla 2. Efectividad de fungicidas sistémicos sobre el crecimiento micelial y la germinación de esporas de Moniliophthora roreri.

Table 2. Effectiveness of systemic fungicides on Moniliophthora roreri mycelial growth and spores germination.

\begin{tabular}{|c|c|c|c|c|}
\hline Fungicida & $\begin{array}{l}\text { Grup } \\
\text { o químico }\end{array}$ & $\begin{array}{c}\text { Dosis } \\
\text { (g.i.a./L) }\end{array}$ & $\begin{array}{c}\text { Inhibición } \\
\text { del } \\
\text { crecimiento } \\
\text { micelialy } \\
(\%) \\
\end{array}$ & $\begin{array}{c}\text { Inhibición } \\
\text { de la } \\
\text { germinación } \\
\text { (\%) }\end{array}$ \\
\hline Testigo & & 0 & $---z^{z}$ & $---z^{z}$ \\
\hline \multirow{4}{*}{ Azoxystrobin } & Estrobirulina & 0.25 & $72.44 \mathrm{~cd}$ & $89.69 c$ \\
\hline & & 0.5 & $73.34 \mathrm{c}$ & $100 \mathrm{a}$ \\
\hline & & 0.75 & $75.58 c$ & $100 \mathrm{a}$ \\
\hline & & 1 & $78.73 b$ & $100 \mathrm{a}$ \\
\hline \multirow{4}{*}{ Trifloxistrobin } & Estrobirulina & 0.25 & $100 \mathrm{a}$ & $100 \mathrm{a}$ \\
\hline & & 0.5 & $100 \mathrm{a}$ & $100 \mathrm{a}$ \\
\hline & & 0.75 & $100 \mathrm{a}$ & $100 \mathrm{a}$ \\
\hline & & 1 & $100 a$ & $100 \mathrm{a}$ \\
\hline \multirow{4}{*}{ Tebuconazole } & Triazol & 0.25 & $100 a$ & $100 a$ \\
\hline & & 0.5 & $100 a$ & $100 a$ \\
\hline & & 0.75 & $100 \mathrm{a}$ & $100 \mathrm{a}$ \\
\hline & & 1 & $100 \mathrm{a}$ & $100 \mathrm{a}$ \\
\hline \multirow{4}{*}{ Propiconazole } & Triazol & 0.25 & $100 \mathrm{a}$ & $98.97 \mathrm{~b}$ \\
\hline & & 0.5 & $100 \mathrm{a}$ & $100 \mathrm{a}$ \\
\hline & & 0.75 & $100 \mathrm{a}$ & $100 \mathrm{a}$ \\
\hline & & 1 & $100 \mathrm{a}$ & $100 \mathrm{a}$ \\
\hline \multirow{4}{*}{ Tiabendazol } & Benzimidazol & 0.25 & $50.98 \mathrm{f}$ & $30.1 \mathrm{~g}$ \\
\hline & & 0.5 & $53.31 \mathrm{f}$ & $47.36 \mathrm{f}$ \\
\hline & & 0.75 & $56.35 \mathrm{f}$ & $55.74 \mathrm{e}$ \\
\hline & & 1 & $59.4 \mathrm{e}$ & $64.12 d$ \\
\hline
\end{tabular}

yMedida con letras iguales dentro de la misma columna, no son estadísticamente diferentes (Tukey, $\mathrm{P}<0.05$ ).

${ }^{2}$ Debido a la naturaleza de la fórmula de Abbott, estos valores no se calcularon.

azoxystrobin y el propiconazole inhibieron la GE al $100 \%$ a partir de 0.5 g.i.a./L (Tabla 2). El fungicida sistémico con menor efectividad sobre la GE fue el tiabendazol.

Todos los fungicidas protectantes inhibieron la GE al $100 \%$ en las dosis evaluadas; sin embargo, hubo diferencias en la efectividad de estos fungicidas sobre el $C M(P<$ 0.0001) (Tabla 3). El sulfato de cobre, hidróxido cúprico, óxido cuproso y oxicloruro de cobre inhibieron el CM al $100 \%$ en las cuatro dosis evaluadas. El polisulfuro de calcio inhibió $100 \%$ la GE y el CM de $M$. roreri en dosis de 8 a 16 g.i.a./L. La mezcla de azufre elemental + oxicloruro de cobre mostró efectividad del $100 \%$ en la inhibición de la GE; sin embargo, su efectividad fue menor al $81 \%$ en la inhibición del CM. El fungicida con menor efectividad sobre el $C M$ de $M$. roreri fue el clorotalonil; empero, este fungicida inhibió al $100 \%$ la GE (Tabla 3). 
Tabla 3. Efectividad de fungicidas protectantes sobre el crecimiento micelial y la germinación de esporas de Moniliophthora roreri.

Table 3. Effectiveness of protectant fungicides on Moniliophthora roreri mycelial growth and spores germination.

\begin{tabular}{|c|c|c|c|c|}
\hline Fungicidas & $\begin{array}{l}\text { Grupo } \\
\text { químico }\end{array}$ & $\begin{array}{c}\text { Dosis } \\
\text { (g.i.a/L) }\end{array}$ & $\begin{array}{c}\text { Inhibición } \\
\text { del } \\
\text { crecimiento } \\
\text { micelialy (\%) }\end{array}$ & $\begin{array}{c}\text { Inhibición } \\
\text { de la } \\
\text { germinación } \\
\text { (\%) }\end{array}$ \\
\hline Testigo & & 0 & $----z$ & $---z$ \\
\hline \multirow{4}{*}{$\begin{array}{l}\text { Sulfato de } \\
\text { cobre } 250\end{array}$} & Cúprico & 0.62 & $100 \mathrm{a}$ & $100 \mathrm{a}$ \\
\hline & & 1.25 & $100 \mathrm{a}$ & $100 \mathrm{a}$ \\
\hline & & 1.87 & $100 a$ & $100 \mathrm{a}$ \\
\hline & & 2.5 & $100 \mathrm{a}$ & $100 \mathrm{a}$ \\
\hline \multirow{4}{*}{$\begin{array}{l}\text { Hidróxido } \\
\text { cúprico }\end{array}$} & Cúprico & 1.87 & $100 \mathrm{a}$ & $100 \mathrm{a}$ \\
\hline & & 3.75 & $100 \mathrm{a}$ & $100 \mathrm{a}$ \\
\hline & & 5.62 & $100 \mathrm{a}$ & $100 a$ \\
\hline & & 7.5 & $100 \mathrm{a}$ & $100 \mathrm{a}$ \\
\hline \multirow{4}{*}{ Óxido cuproso } & Cúprico & 0.93 & $100 a$ & $100 \mathrm{a}$ \\
\hline & & 1.87 & $100 \mathrm{a}$ & $100 \mathrm{a}$ \\
\hline & & 2.81 & $100 \mathrm{a}$ & $100 \mathrm{a}$ \\
\hline & & 3.75 & $100 \mathrm{a}$ & $100 \mathrm{a}$ \\
\hline \multirow{4}{*}{$\begin{array}{l}\text { Oxicloruro de } \\
\text { cobre }\end{array}$} & Cúprico & 1.25 & $100 a$ & $100 \mathrm{a}$ \\
\hline & & 2.5 & $100 \mathrm{a}$ & $100 \mathrm{a}$ \\
\hline & & 3.75 & $100 a$ & $100 \mathrm{a}$ \\
\hline & & 5 & $100 \mathrm{a}$ & $100 \mathrm{a}$ \\
\hline \multirow{4}{*}{$\begin{array}{l}\text { Polisulfuro de } \\
\text { calcio }\end{array}$} & Azufre & 4 & $97.42 \mathrm{~b}$ & $100 \mathrm{a}$ \\
\hline & & 8 & $100 \mathrm{a}$ & $100 \mathrm{a}$ \\
\hline & & 12 & $100 \mathrm{a}$ & $100 \mathrm{a}$ \\
\hline & & 16 & $100 \mathrm{a}$ & $100 \mathrm{a}$ \\
\hline \multirow{4}{*}{$\begin{array}{l}\text { Azufre } \\
\text { elemental + } \\
\text { Oxicloruro de } \\
\text { cobre }\end{array}$} & $\begin{array}{l}\text { Azufre/ } \\
\text { Cúprico }\end{array}$ & $0.45 / 0.04$ & $69.57 d$ & $100 \mathrm{a}$ \\
\hline & & $0.9 / 0.08$ & $79.24 \mathrm{c}$ & $100 a$ \\
\hline & & $1.35 / 0.12$ & 79.25 c & $100 \mathrm{a}$ \\
\hline & & $1.8 / 0.1$ & $80.49 c$ & $100 \mathrm{a}$ \\
\hline \multirow[t]{4}{*}{ Clorotalonil } & Benzonitrilo & 0.25 & $37.55 \mathrm{~g}$ & $100 \mathrm{a}$ \\
\hline & & 0.5 & $60.79 f$ & $100 \mathrm{a}$ \\
\hline & & 0.75 & 64 ef & $100 \mathrm{a}$ \\
\hline & & 1 & $67.21 \mathrm{de}$ & $100 a$ \\
\hline
\end{tabular}

yedida con letras iguales dentro de la misma columna, no son estadísticamente diferentes (Tukey, $\mathrm{P}<0.05$ ).

¿Debido a la naturaleza de la fórmula de Abbott, estos valores no se calcularon.

\section{Efectividad de fungicidas in vivo}

Hubo diferencias en la efectividad de los tratamientos sobre la incidencia natural de la MC $(P<0.0001)$. En el testigo, la incidencia promedio fue del $60 \%$ y la efectividad de los fungicidas varió de 24.2 al $69.96 \%$ en la parcela 1 y de 25.3 al
$72.5 \%$ en la parcela 2 (Figura 1). El tratamiento que incluyó azoxystrobin a 0.8 g.i.a./L, complementado con hidróxido cúprico, fue el de mayor efectividad, sin diferencia con los tratamientos que incluyeron trifloxystrobin ( 0.8 g.i.a./L), tebuconazole ( 0.8 y 0.4 g.i.a./L), propiconazole ( 0.8 y 0.4 g.i.a. $/ \mathrm{L}$ ). El tratamiento con menor efectividad fue el que incluyó azoxystrobin a 0.4 g.i.a./L. Los tratamientos que incluyeron fungicidas del grupo químico de los triazoles, mostraron mayor efectividad a 0.4 g.i.a./L, que aquellos tratamientos que incluyeron estrobirulinas a la misma dosis (Figura 1).

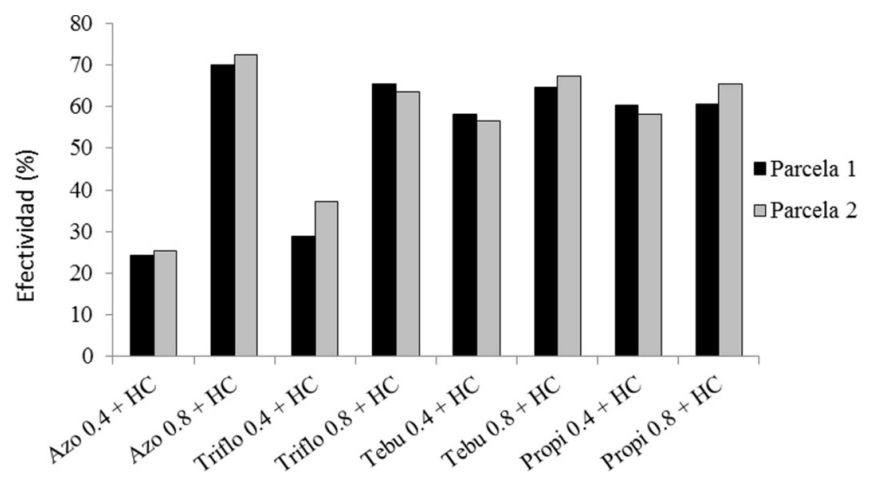

Figura 1. Efectividad de fungicidas sobre la moniliasis del cacao in vivo, a 0.4 y 0.8 g.i.a. L $^{-1}$. Azo $=$ Azoxystrobin, Triflo= Trifloxystrobin, Tebu= Tebuconazole, Propi= Propiconazole, $\mathrm{HC}=$ Hidróxido de cobre a 7.5 g.i.a. $\mathrm{L}^{-1}$. Figure 1. Effectiveness of fungicides on frosty pod rot in vivo, at 0.4 and 0.8 g.i.a. $\mathrm{L}^{-1}$. Azo $=$ Azoxystrobin, Triflo $=$ Trifloxystrobin, Tebu= Tebuconazole, Propi= Propiconazole, $\mathrm{HC}=$ Copper hydroxide at 7.5 g.i.a.. $\mathrm{L}^{-1}$.

\section{DISCUSIÓN}

La importancia de los compuestos químicos que se utilizan para el control de fitopatógenos, radica en su modo de acción sobre la fisiología del patógeno; además de la dosis, frecuencia y etapa de aplicación (Reuveni y Sheglov, 2002). En el presente estudio, M. roreri, aislado del estado de Tabasco, mostró sensibilidad in vitro a todos los fungicidas evaluados, sistémicos y protectantes. Los resultados obtenidos con el azoxystrobin in vitro concuerdan con Torres-de la Cruz et al. (2011) quienes reportaron el $100 \%$ de inhibición del CM y $90 \%$ de inhibición de la GE de M. roreri a una dosis de 0.45 g.i.a./L. La eficacia del trifloxystrobin sobre el CM y la GE de M. roreri se documenta por primera vez. El azoxystrobin y el trifloxystrobin pertenecen al grupo químico de las estrobirulinas; un grupo de fungicidas sistémicos de baja toxicidad (tipo IV) (Thomson, 1997). Estos fungicidas inhiben la respiración mitocondrial deteniendo la producción de ATP, presentan amplio espectro de control y han mostrado efectividad sobre hongos basidiomicetes (Clough y Godfrey, 1996; Bartlett et al., 2002).

El efecto del propiconazol sobre la GE de $M$. roreri concuerda con Ram (1989), el cual reportó $100 \%$ de inhibición de la GE de M. roreri a 100 mg/L. Sin embargo, nuestros resultados difieren con Murillo y González (1984) quienes reportaron un bajo efecto de este fungicida sobre la GE de M. roreri, lo cual pudo deberse a lo bajo de la dosis evaluada (0.01 g.i.a./L.). En nuestro estudio, el propiconazol superó 
el efecto del azoxystrobin, lo cual concuerdan con Aneja et al. (2005), quien reportó que el propiconazol a $1 \mu \mathrm{M}$ mostró mayor inhibición (75\%) del CM de M. roreri que el azoxystrobin (47\%), a la misma dosis. Respecto a la eficiencia del tebuconazole sobre $M$. roreri, esta se reporta por primera vez. El propiconazol y el tebuconazol pertenecen al grupo de los triazoles, un grupo de fungicidas que inhiben el proceso de metilación en la biosíntesis del ergosterol; provocando la ruptura de la membrana celular. De acuerdo con Sheinpflug y Kukc (1987), estos fungicidas son efectivos a bajas concentraciones y han mostrado efectividad sobre basidiomicetes.

El tiabendazol fue el fungicida sistémico que mostró la menor efectividad sobre el DM y la GE de M. roreri, en todas las dosis evaluadas; por lo cual se descartó para la evaluación in vivo. Sin embargo, este fungicida ha mostrado $100 \%$ de efectividad sobre el CM de Fusarium oxysporum, Rhizoctonia solani, y Colletotriichum gloeosporioides a 0.6 g.i.a. / (Gutiérrez y Gutiérrez 2003; López et al. 2005). El tiabendazol pertenece al grupo químico benzimidazol, el cual interfiere en la formación de microtúbulos, necesario para la división celular (Ortiz-Barrera, 1989).

Todos los fungicidas protectantes cúpricos mostraron capacidad de inhibir la GE y el CM de $M$. roreri al $100 \%$ en las dosis evaluadas. De acuerdo con Araúz (1998), los fungicidas a base de cobre son de acción multisitio, de bajo riesgo de resistencia y de baja toxicidad, por lo que constituyen una opción viable en el manejo de la MC. La sensibilidad de $M$. roreri al sulfato de cobre, se documenta por primera vez; sin embargo, este fungicida ha sido ampliamente utilizado para el control de la mancha negra del cacao (Phytophthora capsici en México) (Ortiz-García, 2015). Por otro lado, el hidróxido cúprico es el fungicida protectante que más se ha recomendado para el control de M. roreri en Costa Rica (Hidalgo et al., 2003; Betaman et al., 2005). Este fungicida también ha mostrado efectividad sobre $M$. perniciosa, agente causal de la escoba de bruja, en Brasil (Madeiros et al., 2010). El efecto mostrado por el óxido cuproso, in vitro, concuerda con Ram (1989) quien reportó el $100 \%$ de inhibición de la GE de $M$. roreri a 0.1 g.i.a./L. De acuerdo con Murillo y González (1984), este fungicida mostró una efectividad en campo de $67.2 \%$ sobre M. roreri; así también, Ram (1989) reportó una efectividad en campo del $41 \%$ sobre este patógeno; sin embargo, Murillo y González (1984) reportaron que el óxido cuproso incrementó la incidencia de marchitez fisiológica. En cuanto al oxicloruro de cobre, Ram (1989) reportaron el $100 \%$ de inhibición de la GE de M. roreri, a una dosis de 0.1 g.i.a./L.

El polisulfuro de calcio inhibió la GE y el CM de $M$. roreri al $100 \%$, similar a lo reportado por Ramírez-González et al. (2011). De acuerdo con Williams y Cooper (2004), las células de los hongos pueden absorber azufre, afectando la cadena respiratoria mitocondrial; además, la transferencia de iones de hidrógeno al azufre puede producir sulfito de hidrógeno tóxico. A pesar del efecto mostrado por el polisulfuro de calcio sobre $M$. roreri, se observó crecimiento de $M$. roreri en este fungicida ocho días después de la evaluación, por lo que no se incluyó en la lista de fungicidas potenciales, dado que se requiere confirmar su tiempo de efectividad.

La mezcla de azufre elemental + oxicloruro de cobre mostró efectividad del $100 \%$ en la inhibición de la GE; sin embargo, su efectividad fue menor al 81 \% en la inhibición del CM. Este nivel de efectividad pudo deberse a las dosis utilizadas, debido a que se tomó como referencia la concentración recomendada por el fabricante para frutales. El efecto de esta combinación de fungicidas no ha sido reportado sobre M. roreri.

El fungicida protectante con menor efectividad sobre el CM de $M$. roreri fue el clorotalonil; sin embargo, este fungicida inhibió al $100 \%$ la GE. Lo anterior concuerda con Ram (1989), el cual reportó inhibición de la GE de M. roreri a partir de 0.001 g.i.a./L. Por su parte, Murillo y González (1984) reportaron un $54 \%$ de efectividad de este fungicida sobre $M$. roreri, bajo condiciones de Campo, en Costa Rica. El clorothalonil es un fungicida de contacto del grupo benzonitrilo, el cual actúa interfiriendo el metabolismo energético de la célula (Arauz, 1998). Este fungicida se destaca por su persistencia y tolerancia al lavado de lluvias y riego y es efectivo contra una amplia gama de hongos fitopatógenos. Sin embargo, de acuerdo con Murillo y Gonzales (1984) y Cox (1997), el clorotalonil es tóxico a los operadores (clase I y II) de acuerdo con la World Health Organization (WHO)/EPA.

En frutos jóvenes infectados naturalmente en campo, el efecto del azoxystrobin ( 0.8 g.i.a./L) complementado con hidróxido de cobre (7.5 g.i.a/L) concuerda con Torres-de la Cruz et al. (2011) y Ortiz-García et al. (2015). Sin embargo, en este trabajo también se demuestra la eficiencia de la aplicación temprana de trifloxystrobin, tebuconazol o propiconazol, en frutos de hasta dos meses de edad, complementado con aplicaciones posteriores de hidróxido de cobre. Lo anterior concuerda con Bateman et al. (2005) quienes recomiendan la aplicación del fungicida sistémico flutolanil, en los primeros 60 días de desarrollo del fruto de cacao, seguido de aplicaciones mensuales con hidróxido de cobre, para el control de la MC. Esta estrategia también coincide con Carmona (2010) quien recomienda reservar la utilización de los fungicidas sistémicos a fases críticas del desarrollo del cultivo o etapa fenológica; además, el uso de fungicidas con diferentes modos de acción, puede disminuir el riesgo de desarrollo de resistencia (Rouveni y Sheglov, 2002).

\section{CONCLUSIONES}

Moniliophthora roreri, aislado del estado de Tabasco, México, presentó sensibilidad a los fungicidas sistémicos azoxystrobin, trifloxystrobin, tebuconazole y propiconazole. Moniliophthora roreri también es sensible a los fungicidas protectantes sulfato de cobre, hidróxido de cobre, óxido cuproso, oxicloruro de cobre y polisulfuro de calcio. Los fungicidas azoxystrobin, clorotalonil y la mezcla azufre elemental + oxicloruro de cobre presentaron mayor efectividad sobre la GE que en el CM. En frutos jóvenes (chilillos) con infección natural de campo, una aplicación de azoxystrobin, trifloxys-

Volumen XXI, Número 2 
trobin, tebuconazol o propiconazol ( 0.8 g.i.a./L), complementada con aplicaciones mensuales de hidróxido de cobre (7.5 g.i.a./L) por tres meses, disminuyó significativamente la incidencia de la moniliasis del cacao. La sensibilidad in vitro de $M$. roreri, a los fungicidas sulfato de cobre, oxido cuproso y oxicloruro de cobre, abre la posibilidad de probarlos en campo en futuras investigaciones.

\section{AGRADECIMIENTOS}

Los autores agradecen al CONACYT por los recursos otorgados para el desarrollo de esta investigación (CB-200801-106570).

\section{REFERENCIAS}

Abbott, W.S. 1925. A method of computing the effectiveness of an insecticide. Journal of Economic Entomology. 18: 256267.

Aime, M.C.y Phillips-Mora, W. 2005. The causal agents of witches' broom and frosty pod rot of cacao (chocolate, Theobroma cacao) form a new lineage of Marasmiaceae. Mycology. 97: 1012-1022.

Amaya, L., Bustamante, E., Navarro, L. y Hernández, A. 1976. Estudio histopatológico de mazorcas de cacao (Theobroma cacao L.) infectados con el hongo Monilia roreri Cif y Par. Noticias Fitopatológicas (Colombia). 5: 97-98.

Ampuero, C.E. 1967. Monilia pod rot of cocoa. Cocoa Growers' Bulletin. 9: 15-18.

Aneja, M., Gianfagnaa, T.J. y Hebbarb, P.K. 2005. Trichoderma harzianum produces nonanoic acid, an inhibitor of spore germination and mycelial growth of two cacao pathogens. Physiological and Molecular Plant Pathology. 67: 304-307.

Arauz, L. 1998. Fitopatología: un enfoque agroecológico. Universidad de Costa Rica. Costa Rica.

Bartlett, D.W., Clough, J.M., Godwin, J.R., Hall, A.A., Hamer, M. y Parr-Dobrzansk, B. 2002. The strobilurin fungicides. Pest Management Science. 58: 649-662.

Barros, N.O. 1966. Valor de las prácticas culturales como método para reducir la incidencia de Monilia en plantaciones de cacao. Agricultura Tropical. 22: 605-612.

Bateman. R.P., Hidalgo, E., García, J., Arroyo, C., ten Hoopen, G.M., Adonijah, V. y Krauss, U. 2005. Application of chemical and biological agents for the management of frosty pod rot (Moniliophthora roreri) in Costa Rican cocoa (Theobroma cacao). Annals of Applied Biology. 147: 129-138.

Clough, J.M., y Godfrey, C.R.A. 1996. Azoxystrobin: A novel broad-spectrum systemic fungicide. Pesticide Outlook 7:1620.

Cox, C. 1997. Chlorothalonil. Journal of Pesticide Reform, 17: 14-20.

Galindo, J.J. 1984. Programa de cacao investiga moniliasis. Actividades en Turrialba CATIE. 12: 8-9.

González, L.C., Sánchez, J.A., Porras, V.H., Umaña, S. y Murillo, D. 1983. Evaluación del fungicida clorotalonil y de la destrucción de mazorcas enfermas en el combate de la moniliasis del cacao. Agronomía Costarricense. 7: 1-7.

Griffith, W.G., Nicholson, J., Nenninger, A., Birch, N.R. y Hedger, N.J. 2003. Witches' brooms and frosty pods: two major pathogens of cacao. New Zealand Journal of Botany. 41: 423-435.
Gutiérrez-Alonso, O. y Gutiérrez-Alonso, J.G. 2003. Evaluación de resistencia a Benomil, Thiabendazol y Azoxystrobin, para el control de antracnosis (Colletotrichum gloeosporioides PENZ.) en frutos de Guayaba (Psidium guayava L.) en postcosecha. Revista Mexicana de Fitopatología. 21:228-232.

Hidalgo, E., Bateman, R.P., Krauss, U., ten Hoopen, M. y Martínez, A. 2003. A field investigation into delivery systems for agents to control Moniliophthora roreri. European Journal of Plant Pathology. 109: 953-961.

Krauss, U. y Soberanis, W. 2002. Effect of fertilization and biocontrol application frequency on cocoa pod diseases. Biological control. 24: 82-89.

Krauss, U., ten Hoopen, M., Hidalgo, E., Martínez, A., Arroyo, C., García, J., Portugués, A. y Sánchez, V. 2003. Manejo integrado de la moniliasis (Moniliophthora roreri) del cacao (Theobroma cacao) en Talamanca, Costa Rica. Agroforestería en las Américas. 10: 52-58.

López, B.A., López, B.S.R., Vázquez, B.M.E., Rodríguez, H.S.A., Mendoza, E.M. y Padrón, B.C. 2005. Inhibición del crecimiento micelial de Fusarium oxysporum Schlechtend F. sp. Lycopersici (sacc.) Snyder y Hansen, Rhizoctonia solani Kühn y Verticillium dahliae Kleb, mediante extractos. Revista Mexicana de Fitopatología. 23: 183-190.

Medeiros, F.H.V., Pomella, A.W.V., De Souza, J.T., Niella, G.R., Valler, R.P., Bateman, R.P., Fravel, D., Vinyard, B. y Hebbar, P.K. 2010. A novel, integrated method for management of witches' broom disease in Cacao in Bahia, Brazil. Crop Protection. 29: 704-711.

Murillo, D. y González, L.C. 1984. Evaluación en laboratorio y campo de fungicidas para el combate de la moniliasis del cacao. Agronomía Costarricense. 8: 83-89.

Ortiz-Barrera, R. 1989. Manejo de la resistencia a Fungicidas. CIBA-GEIGY. México.

Ortiz-García, C.F., Torres-de la Cruz, M. y Hernández-Mateo, S. 2015. Comparación de dos sistemas de manejo del cultivo del cacao, en presencia de Moniliophthora roreri, en México. Revista Fitotecnia Mexicana. 38: 191-196.

Phillips-Mora, W., Castillo, J., Krauss, U., Rodriguez, E. y Wilkinson, J. 2005. Evaluation of cacao (Theobroma cacao) clones against seven Colombian isolates of Moniliophthora roreri from four pathogen genetic groups. Plant Pathology. 54: 483-490.

Ram, A. 1989. Biology, epidemiology and control of moniliasis (Moniliophthora roreri) of cacao. University of London. London.

Ramírez-González, S., López-Báez, O., Guzmán-Hernández, T., Munguía-Ulloa, S. y Moreno-Martínez, J.L. 2011. El polisulfuro de calcio en el manejo de la moniliasis Moniliophthora roreri (Cif \& Par). Evans et al. del cacao Theobroma cacao L. Tecnología en Marcha. 24:10-18.

Reuveni, M. y Sheglov, D. 2002. Effects of azoxystrobin, difeconazole, polyoxin B (polar) and Trifloxystrobin on germination and growth of Alternaria alternata and decay in red delicious apple fruit. Crop Protection, 21: 951-955.

SAS Institute Inc. 1988. SAS User's Guide: Statistics, Release 6.03. Ed. SAS Institute INC. Cary, NC.

Sheinpflug, H. y Kukc, K.W. 1987. Sterol biosynthesis inhibiting piperazine, pyredine, pyrimidine y azole fungicides en: Modern selective fungicide properties, applications, Mechanics of action. H. Lyr (ed.), pp. 173-204. Longman group. UK, London. 
Thomson, W.T. 1997. Agricultural Chemicals. Book IV: Fungicides. 12th edition. Thomson Publications, Fresno, CA.

Tirado-Gallego, P.A., Lopera-Álvarez, A., Ríos-Osorio, L.A. 2016. Estrategias de control de Moniliophthora roreri y Moniliophthora perniciosa en L.: revisión sistemática. Corpoica Cienc Tecnol Agropecuaria, 17: 417-430.

Torres-de la Cruz, M., Ortiz-García, C.F., Teliz-Ortiz, D., MoraAguilera, A. y Nava-Díaz, C. 2011. Temporal progress and integrated management of frosty pod rot [Moniliophthora roreri (Cify Par.) Evans et al.] of cocoa (Theobroma cacao) in Tabasco, Mexico, Journal of Plant Pathology. 93: 31-36.
Torres-de la Cruz, M., Ortiz-García, F.F., Nava-Díaz, C. y de la CruzPérez, A. 2016. Confirmación morfológica y molecular de Moniliopthora roreri, agente causal de la moniliasis del cacao (Theobroma cacao) en Tabasco, México. En: Perspectiva Científica desde la UJAT. W.M. Contrera-Sanchez (ed.), p. 407-412. Universidad Juárez Autónoma de Tabasco. México. Williams, J.S. y Cooper, R.M. 2004. The oldest fungicide and newest phytoalexin - a reappraisal of the fungitoxicity of elemental sulphur. Plant Pathology 53: 263- 279. 